\title{
REDUCCIÓN DEL TIEMPO DE FINALIZACIÓN DEL PROYECTO DE UNA PLANTA DE CONSERVAS DE PESCADO UTILIZANDO UN MODELO DE PROGRAMACIÓN LINEAL
}

\author{
REDUCING THE TIME OF COMPLETION OF THE PROJECT IN A FISH CANNING PLANT \\ USING A LINEAR PROGRAMMING MODEL
}

\author{
Christian R. Ramos A. ${ }^{1}$ y Carlos A. Flores B. ${ }^{2}$
}

\begin{abstract}
Resumen
En el presente trabajo de investigación se utilizó el método de la ruta crítica en donde se calculó el tiempo de finalización del proyecto y la ruta crítica de un proyecto de inversión para la instalación de una planta de conservas de anchoveta. El objetivo es determinar las reducciones del tiempo de finalización del proyecto de inversión para minimizar el costo adicional por reducción. El tiempo de finalización del proyecto determinado es de 96 días, las actividades no críticas está conformada por los trabajos preliminares, instalaciones sanitarias, instalaciones eléctricas e instalación de máquinas, es decir tienen holgura para demorar en iniciar, el resto de actividades conforman la ruta crítica.
\end{abstract}

Se construyó un modelo de programación lineal y la solución redujo el tiempo de finalización del proyecto hasta 55 días con un costo adicional por reducción de S/. 47,412.

Palabras clave: Diagrama de Gantt, PERT/CPM, programación lineal, reducción del tiempo, ruta crítica.

\begin{abstract}
In the present research work the critical path method where time of completion of the project was calculated and the critical path of an investment project for the installation of a plant of canned anchovy was used. The objective is to determine the completion time reductions of the investment project to minimize the additional cost reduction. The completion time for the given project is 96 days, the noncritical activities consist of the preliminary work, plumbing, wiring and installation of machines, that is to say that they have clearance to delay in starting the other activities that constitute the critical path.
\end{abstract}

A linear programming model was constructed and the solution reduced the time of completion of the project up to 55 days with an additional cost reduction of $\mathrm{S} / .47,412$.

Keys words: Critical path, Gantt chart, linear programming, PERT/CPM, reducing the time.

\section{Introducción}

En la administración de proyectos se realizan los cálculos para determinar el tiempo de finalización del proyecto y encontrar la ruta crítica, utilizando dos métodos de modelos de redes llamados: método de la ruta crítica ó CPM (del inglés Critical Path Method) y la técnica de evaluación y revisión del programa ó PERT (del inglés Programme Evaluation Review Technique) (Mehrotra et al., 1996).

Al calcular el tiempo de finalización del proyecto se determinan los tiempos más tempranos y más tardíos de cada actividad y posteriormente se podrá realizar la programación de actividades a través de un diagrama de Gantt (Taha, 2004).
En muchos casos se requiere terminar el proyecto antes de la fecha establecida, es por ello que se desea saber qué actividades se deben de reducir y en cuanto (Anderson et al., 2004; Render et al., 2006), para eso se construye un modelo de programación lineal para responder a la pregunta. La forma genérica del modelo podrá ser extrapolado a otros casos particulares (Winston, 2005; Hillier, 2006).

El objetivo del presente trabajo de investigación es determinar las reducciones del tiempo de finalización de un proyecto de inversión de conservas de pescado para minimizar el costo adicional por reducción.

\footnotetext{
${ }^{1}$ Facultad de Pesquería. Universidad Nacional Agraria La Molina. Lima, Perú. E-mail: cramos@lamolina.edu.pe

${ }^{2}$ Facultad de Ingeniería Industrial y de Sistemas. Universidad Nacional de Ingeniería. Lima, Perú. E-mail: cflores@uni.edu.pe
} 


\section{Materiales y métodos}

\subsection{Inversión de la planta de conservas}

El cálculo de la inversión de la planta de conservas seguirá la siguiente secuencia, primero se definirá el proceso de elaboración de las conservas, ello nos llevará a determinar los requerimientos de máquinas $\mathrm{y}$ equipos en las operaciones unitarias del proceso y calcular su inversión. Posteriormente, y considerando las dimensiones de los equipos, se propone un diseño de planta que incluya la sala de proceso, almacenes, oficinas, y otras áreas de servicio, con ello se estiman los costos para la construcción de las instalaciones el cuál se dividirá en actividades que estén interrelacionadas con sus respectivas duraciones en días.

\subsection{Aplicación del método de la ruta crítica (CPM)}

En la administración de proyectos se utilizan muchas herramientas que nos permiten realizar representaciones gráficas y cálculos, como son los grafos, diagramas de Gantt para el cronograma de actividades y las herramientas cuantitativas CPM (Método de la ruta crítica) y PERT (Técnica de evaluación y revisión del programa).

Los métodos CPM y PERT tienen un procedimiento similar y se diferencian en las duraciones de las actividades, mientras que el primero considera tiempos determinísticos, un solo tiempo por actividad, el segundo utiliza tiempos probabilísticos, tres tiempos por cada actividad (optimista, más probable o normal y pesimista)

En el método CPM, la red del proyecto se construye a partir de la duración $t_{i}$ de cada actividad $i$, que viene a ser una representación gráfica o grafo en donde se muestran las actividades dentro de nodos, la relación de precedencia entre las actividades, unidas por arcos dirigidos o flechas, cuyas actividades iniciales están a la izquierda y las finales a la derecha.

Luego de construir la red del proyecto se realizan los cálculos de la red determinando primero los tiempos más tempranos, inicio más temprano $E S_{i}$ y finalización más temprana $E F_{i}$ y posteriormente los tiempos más tardíos, inicio más tardío $L S_{i}$ y finalización más tardía $L F_{i}$.

En las ecuaciones (1) y (2) se muestran los cálculos de los tiempos de finalización más temprana $E F_{i}$ e inicios más tardío $L S_{i}$ de cada actividad $i$ respectivamente.

$E F_{i}=E S_{i}+t_{i}$

$L S_{i}=L F_{i}-t_{i}$

La ruta crítica está conformada por las actividades críticas que son aquellas que no tienen holgura $H_{i}$ o es igual a cero.

El cálculo de la holgura de cada actividad $i$ se muestra en la ecuación (3)

$H_{i}=L S_{i}-E S_{i}$

El tiempo de finalización del proyecto Tproy viene a ser el tiempo de finalización más temprana de la última actividad en terminar.

El programa de actividades resume lo realizado en los cálculos de la red y el diagrama de Gantt representa por medio de un gráfico de barras horizontales los inicios, terminaciones y duraciones de las actividades y sus interrelaciones.

\subsection{Reducción del tiempo de finalización del proyecto}

La reducción del tiempo de finalización del proyecto se realiza agregando más recursos a las actividades para acortar la duración de ellas.

La ecuación (4) representa la reducción máxima del tiempo $M_{i}$ como la diferencia del tiempo esperado $t_{i}$ de la actividad $i$ menos el tiempo de acortamiento máximo $t$, de la actividad $i$.

$M_{i}=t_{i}-t_{i}^{\prime}$

La ecuación (5) representa el costo de reducir por unidad de tiempo $K_{i}$ como la razón de la diferencia de los costos de reducción máxima $C^{\prime}{ }_{i}$ y el costo a tiempo normal $C_{i}$ entre la reducción máxima del tiempo $M_{i}$ de la actividad $i$.

$K_{i}=\left(C_{i}{ }^{\prime}-C_{i}\right) / M_{i}$

La reducción del tiempo de finalización del proyecto se realiza construyendo un modelo de programación lineal que estará conformado por una función objetivo de minimización y las restricciones.

La función objetivo consiste en minimizar el costo adicional $K_{i}$ por reducción de tiempo $Y_{i}$ de las actividades $i=1$ a la $n$, tal como se muestra en la ecuación (6)

Minimizar $\mathrm{Z}=\sum_{(\mathrm{i}=1)}^{\mathrm{n}} Y_{\mathrm{i}} K_{i}$

En la inecuación (7) se plantea el cálculo del tiempo de finalización más temprano $X_{i}$ de cada actividad $i$ considerando el tiempo de reducción $Y_{i}$.

$X_{i} \geq X_{(i-1)}+\left(t_{i}-Y_{i}\right)$

$X_{i}+Y_{i}-X_{(i-1)} \geq t_{i}, \quad \forall i=1, \ldots, \mathrm{n}$

El tiempo de finalización más temprana de la última 
actividad en terminar $X_{n}$ es menor igual a un tiempo preestablecido $T_{\text {pre }}$ que debe ser menor al tiempo de finalización del proyecto $T_{\text {proy }}$ y a la vez deberá pertenecer a los números enteros positivos, tal como se observa en (8).

$$
X_{n} \leq T_{\text {pre }}, \quad \forall T_{\text {pre }}<T_{\text {proy }} \wedge T_{\text {pre }} \in \mathbb{Z}^{+}
$$

En (9) se muestra la restricción de que el tiempo de reducción $Y_{i}$ de cada actividad $i$ debe de ser menor igual al tiempo de reducción máxima $M_{i}$.

$Y_{i} \leq M_{i}, \quad \forall i=1, \ldots, n$

La restricción de no negatividad de las variables $X_{i}$ e $Y_{i}$ se observa en (10).

$$
Y_{i} \geq 0 \wedge \wedge_{\mathrm{Y}^{\prime}} \geq 0, \quad \forall i=1, \ldots, \mathrm{n}
$$

Se utilizó el software QM for Windows 4 para la solución del modelo de programación lineal.

\section{Resultados y discusión}

\subsection{Inversión de la planta de conservas}

El proyecto de inversión consiste en instalar una planta elaboradora de conservas de anchoveta en salsa de tomate y el proceso de producción se muestra en el diagrama de operaciones de procesos (DOP) de la Fig. 1, el cual consta de dos subprocesos, uno para elaborar la salsa de tomate el cual se une al subproceso de transformación de la anchoveta en tubo en conservas de pescado.

El peso escurrido del producto es de 120 gr y con 60 gr de salsa de tomate como líquido de gobierno a $14^{\circ}$ Brix.

En la Fig. 1 se mostró el DOP de la elaboración de conservas de anchoveta, el cual está conformado por operaciones unitarias que permiten la transformación de las materias primas e insumos en el producto final. En cada operación unitaria se transforma estos por medio de máquinas y equipos. En la Tabla 1 se detalla las máquinas, equipos y recipientes a utilizar en la planta de conserva de anchoveta, sus cantidades, costos unitarios y la inversión en ellas. Es importante resaltar que el costo unitario en las máquinas y equipos incluye la instalación de estos.

\section{Pasta concentrada de tomate}

$30^{\circ} \mathrm{Brix}$

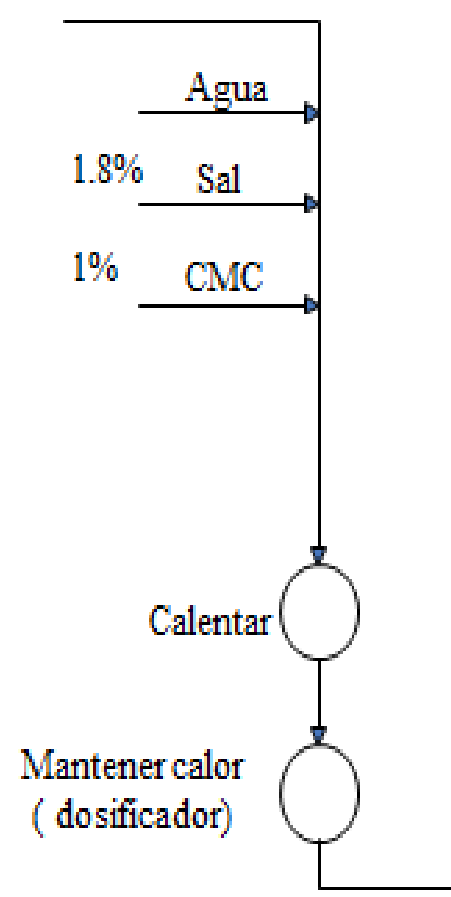

Anchoveta en tubo

\section{Salsa de Tomate}

$14^{\circ} \mathrm{Brix}$

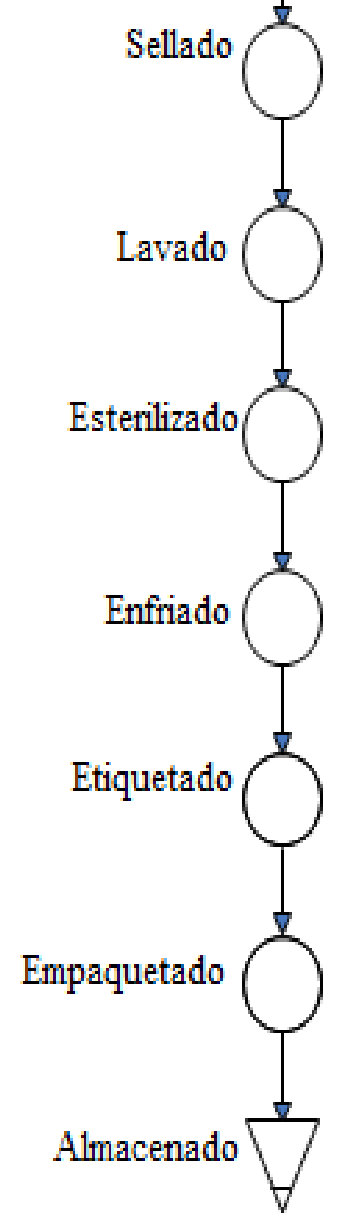

Figura 1. Diagrama de operaciones del proceso de Elaboración de conservas de anchoveta. 
Tabla 1. Inversión en máquinas, equipos y recipientes de la planta de conserva de anchoveta.

\begin{tabular}{llll}
\hline \multicolumn{1}{c}{ Concepto } & Cantidad $\begin{array}{c}\text { Costo } \\
\text { unitario } \\
(\mathrm{S} / .)\end{array}$ & $\begin{array}{c}\text { Inversión } \\
(\mathrm{S} / .)\end{array}$ \\
\hline Mesa de envasado & 1 & 5,000 & 5,000 \\
Cinta transportadora & 2 & 3,000 & 6,000 \\
Cocinador continuo & 1 & 30,000 & 30,000 \\
Marmita & 2 & 13,000 & 26,000 \\
Dosificador & 1 & 6,000 & 6,000 \\
Cerradora de latas & 1 & 30,000 & 30,000 \\
Lavadora & 1 & 5,000 & 5,000 \\
Autoclave & 2 & 40,000 & 80,000 \\
Balanza & 1 & 800 & 800 \\
Balanza electrónica & 1 & 200 & 200 \\
Carritos para autoclave & 6 & 300 & 1,800 \\
Dynos & 10 & 300 & 3,000 \\
Carretilla hidráulica & 1 & 1,000 & 1,000 \\
Pallets & 40 & 30 & 1,200 \\
Caldero & 1 & 100,000 & 100,000 \\
Bomba & 3 & 700 & 2,100 \\
Tanque de petróleo & 1 & 3,000 & 3,000 \\
Tanque de agua & 1 & 2,000 & 2,000 \\
\hline Total & & & 303,100 \\
\hline
\end{tabular}

Luego de calcular los requerimientos de máquinas y equipos, se propone un diseño de planta que considere dimensiones y espacios a ocupar, así como otras instalaciones como almacén, oficinas, laboratorio, servicios higiénicos y espacios para la cisterna, tanques de petróleo y de agua, tal como se muestra en la Fig. 2. En la implementación de la planta, se consideran actividades de obras civiles como las de edificaciones, estructuras, arquitectura, instalaciones sanitarias e instalaciones eléctricas y mecánicas.

En la Tabla 2 se detalla el costo a tiempo normal $C i$ de cada actividad $i$, tanto de las obras civiles como el de instalación de las máquinas.

Tabla 2. Costo a tiempo normal de las actividades del proyecto de conserva de anchoveta.

\begin{tabular}{ccc}
\hline Actividad & Descripción & $\begin{array}{c}\text { Costo a tiempo } \\
\text { normal en S/. } \\
(\mathrm{C} i)\end{array}$ \\
\hline A & Obras provisionales & 4,403 \\
B & Trabajos preliminares & 5,788 \\
C & Movimiento de tierras & 17,744 \\
D & Cimentación & 88,552 \\
E & Columnas y vigas & 98,292 \\
F & Instalaciones sanitarias & 29,172 \\
G & Instalaciones eléctricas & 14,327 \\
H & Muros & 40,996 \\
I & Revestimiento & 46,048 \\
J & Instalación de máquinas & 303,100 \\
K & Techo & 32,073 \\
L & Acabados & 115,442 \\
\hline & & 795,937 \\
\hline
\end{tabular}

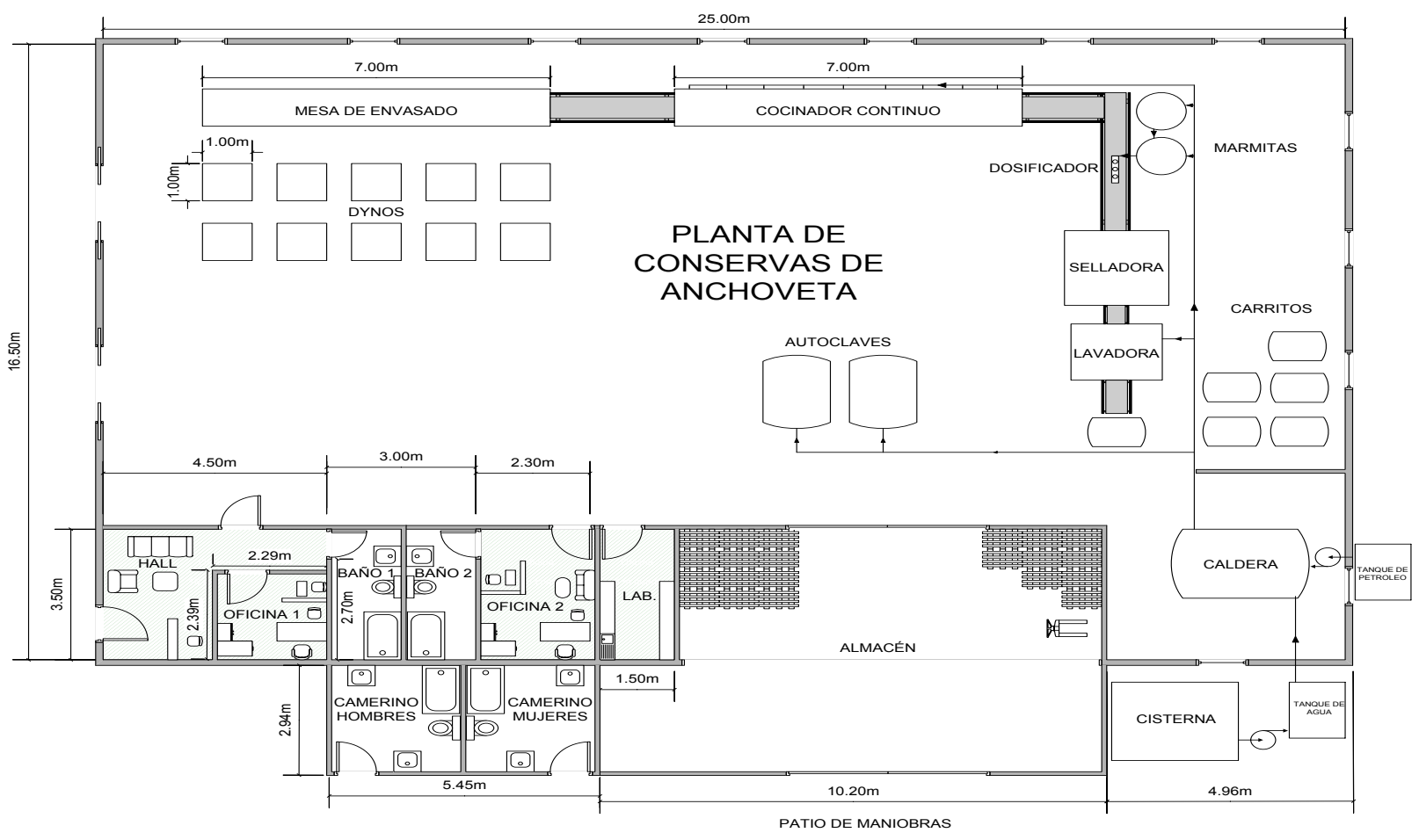

Figura 2. Plano de la planta de conservas de anchoveta. 


\subsection{Método de la ruta crítica (CPM)}

Una vez que se defina las actividades que constituirá el proyecto, se establece la relación de predecesoras inmediatas que hay entre ellas tal como se observa en la Tabla 3 .

Tabla 3. Descripción y predecesoras inmediatas de las actividades.

\begin{tabular}{cccc}
\hline \multirow{2}{*}{ Actividad } & \multirow{2}{*}{ Descripción } & $\begin{array}{c}\text { Predecesora } \\
\text { inmediata }\end{array}$ & $\begin{array}{c}\text { Duración } \\
\text { (días) }\end{array}$ \\
\hline A & Obras provisionales & - & 15 \\
B & Trabajos preliminares & - & 10 \\
C & Movimiento de tierras & A,B & 5 \\
D & Cimentación & $\mathrm{C}$ & 12 \\
E & Columnas y vigas & D & 10 \\
F & Instalaciones sanitarias & $\mathrm{D}$ & 15 \\
G & Instalaciones eléctricas & $\mathrm{D}$ & 13 \\
H & Muros & $\mathrm{E}$ & 15 \\
I & Revestimiento & $\mathrm{F}, \mathrm{H}$ & 7 \\
J & Instalación de máquinas & $\mathrm{G}$ & 20 \\
K & Techo & $\mathrm{I}$ & 17 \\
L & Acabados & $\mathrm{J}, \mathrm{K}$ & 15 \\
\hline
\end{tabular}

Las actividades iniciales son aquellas que no tienen predecesoras inmediatas como son las obras provisionales y los trabajos preliminares, y las actividades finales son aquellas que no son predecesoras inmediatas de otras, como son los acabados de la obra.

En la Fig. 3 se muestra la red del proyecto construida a partir de las predecesoras inmediatas, cada nodo circular representa a cada actividad y las flechas la interrelación que hay entre ellas. En la parte superior de cada nodo se encuentra la duración de cada actividad en días.

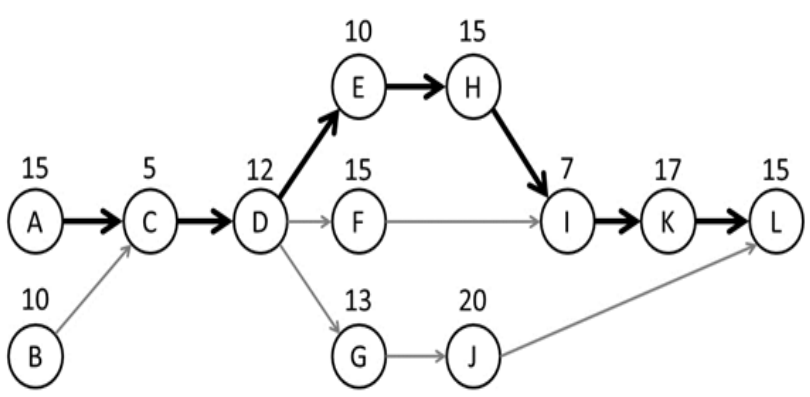

Figura 3. Red del proyecto con las duraciones estimadas de cada actividad.

En los cálculos de la red utilizando las ecuaciones (1) y (2), presentados en la Fig. 4, se observan los tiempos más tempranos (parte superior de los nodos), los tiempos más tardíos (parte inferior de los nodos), los inicios (lado izquierdo de los nodos) y las finalizaciones (lado derecho de los nodos).

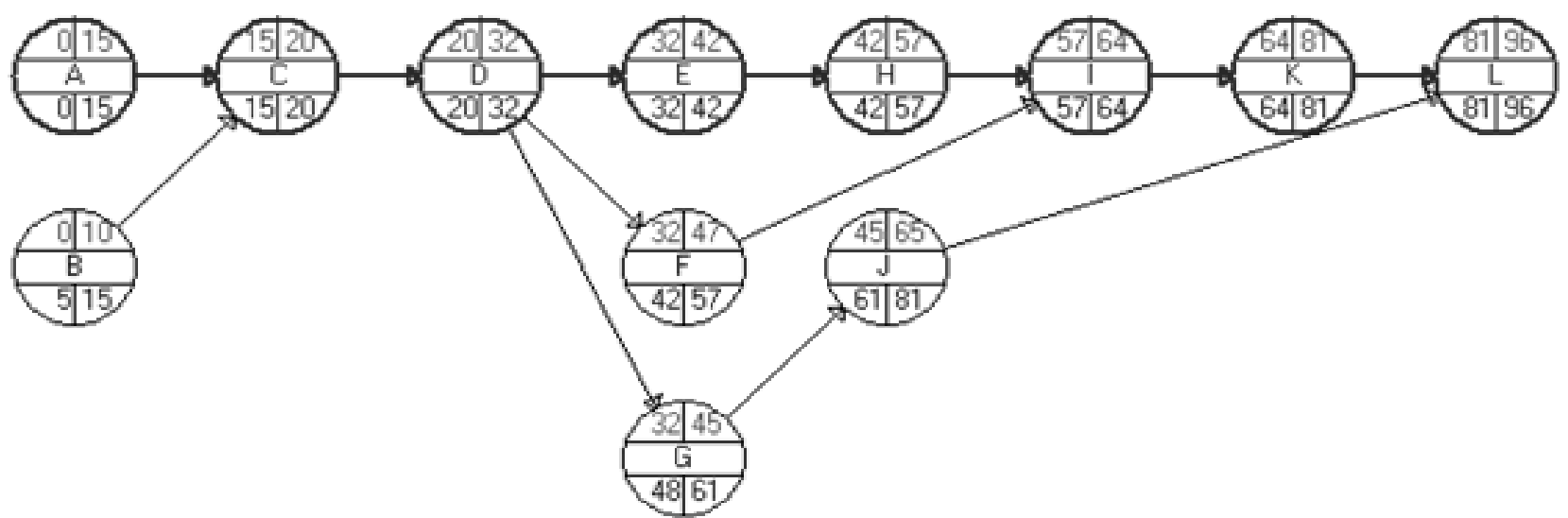

Figura 4. Red del proyecto con sus tiempos más tempranos y más tardíos.

El tiempo de finalización del proyecto estimado es de 96 días y la ruta crítica está conformada por las actividades A, C, D, E, H, I, K y L.

El programa de actividades del proyecto es el resumen de los cálculos de la red, se muestran para cada actividad, su duración, los inicios más tempranos $(E S)$, finalizaciones más tempranas $\left(E F_{\mathrm{i}}\right)$, inicios más tardíos $\left(L S_{i}\right)$, finalizaciones más tardías $\left(L F_{\mathrm{i}}\right)$ y las holguras de cada actividad $\left(H_{\mathrm{i}}\right)$ calculadas con la ecuación (3) y que se presentan en la Tabla 4.
Tabla 4. Programa de actividades del proyecto.

\begin{tabular}{lllllll}
\hline Actividad & $\begin{array}{c}\text { Duración } \\
\text { (días) }\end{array}$ & $E S_{i}$ & $E F_{i}$ & $L S_{i}$ & $L F_{i}$ & $H_{i}$ \\
\hline $\mathrm{A}$ & 15 & 0 & 15 & 0 & 15 & 0 \\
$\mathrm{~B}$ & 10 & 0 & 10 & 5 & 15 & 5 \\
$\mathrm{C}$ & 5 & 15 & 20 & 15 & 20 & 0 \\
$\mathrm{D}$ & 12 & 20 & 32 & 20 & 32 & 0 \\
$\mathrm{E}$ & 10 & 32 & 42 & 32 & 42 & 0 \\
$\mathrm{~F}$ & 15 & 32 & 47 & 42 & 57 & 10 \\
$\mathrm{G}$ & 13 & 32 & 45 & 48 & 61 & 16 \\
$\mathrm{H}$ & 15 & 42 & 57 & 42 & 57 & 0 \\
$\mathrm{I}$ & 7 & 57 & 64 & 57 & 64 & 0 \\
$\mathrm{~J}$ & 20 & 45 & 65 & 61 & 81 & 16 \\
$\mathrm{~K}$ & 17 & 64 & 81 & 64 & 81 & 0 \\
$\mathrm{~L}$ & 15 & 81 & 96 & 81 & 96 & 0 \\
\hline
\end{tabular}


Enero - Junio 2016

La Tabla 4 también muestra que las actividades críticas son aquellas que no tienen holgura es decir son iguales a cero, es decir que no pueden demorar en sus inicios para que el proyecto termine en 96 días.

Las actividades no críticas, es decir las que tienen holgura, son las instalaciones sanitarias $(\mathrm{F})$, instalaciones eléctricas $(\mathrm{G})$ y la instalación de las máquinas $(\mathrm{J})$, eso significa que pueden demorar en su inicio hasta 10, 16 y
16 días respectivamente para que el proyecto termine en 96 días, sin embargo si demoran en iniciar en un tiempo mayor a sus holguras, el proyecto tendrá atrasos en su culminación.

En la Fig. 5 se observa el diagrama de Gantt del proyecto, en el eje de las abscisas está representado el tiempo en días y el eje de las ordenadas representa a las actividades del proyecto.

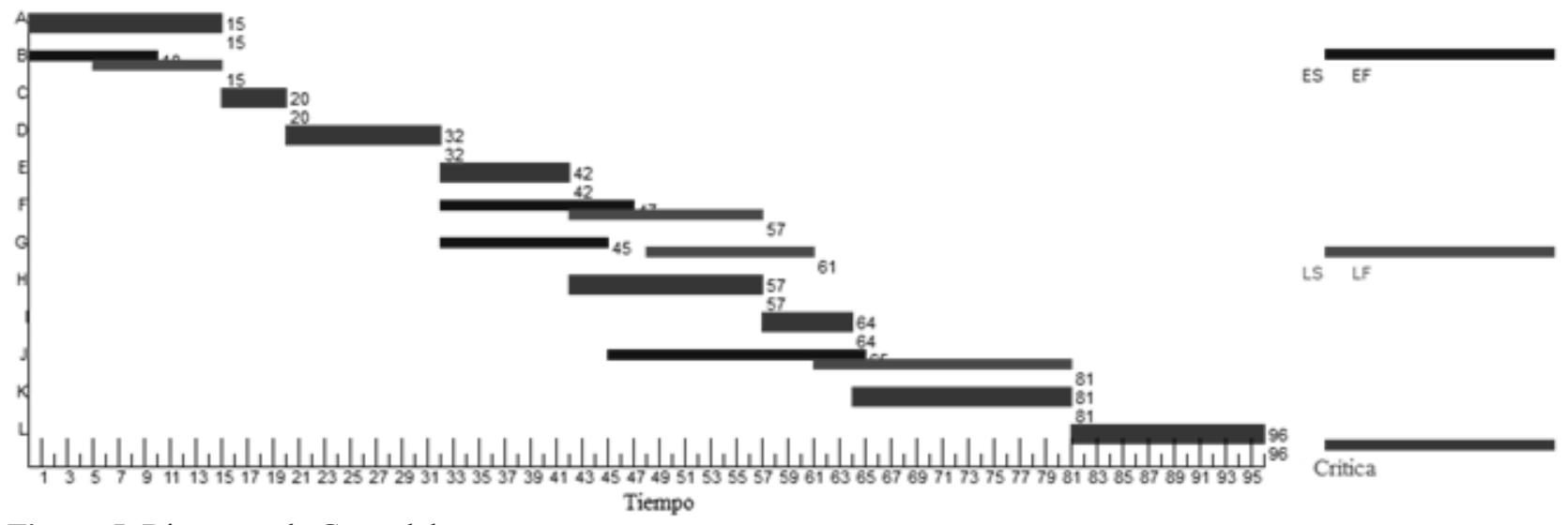

Figura 5. Diagrama de Gantt del proyecto.

\subsection{Reducción del tiempo de finalización del proyecto.}

En la Tabla 5 se presentan los tiempos normales y de reducción máxima de cada actividad, así como también los costos a tiempo normal y de reducción máxima.
En los costos de reducción se consideró un incremento del $20 \%$ del costo de la mano de obra de cada partida de construcción debido a un incremento en la mano de obra y por consiguiente en la reducción de las duraciones de cada actividad.

Tabla 5. Resumen de costos por cada actividad a tiempo normal y reducida para el proyecto de conservas de anchoveta.

\begin{tabular}{|c|c|c|c|c|c|c|c|}
\hline \multirow[b]{2}{*}{ Actividad } & \multirow[b]{2}{*}{ Descripción } & \multicolumn{2}{|c|}{ Tiempo (días) } & \multicolumn{2}{|c|}{ Costos $(\mathrm{S} /)}$. & \multirow{2}{*}{$\begin{array}{c}\text { Reducción } \\
\text { máxima en días } \\
(M i)\end{array}$} & \multirow{2}{*}{$\begin{array}{l}\text { Costo de } \\
\text { reducir } \mathrm{S} / \text {. } \\
\text { por día }(K i)\end{array}$} \\
\hline & & Normal & Reducción & $\begin{array}{c}\text { Normal } \\
(C i)\end{array}$ & $\begin{array}{l}\text { Reducción } \\
\qquad\left(C^{\prime} i\right)\end{array}$ & & \\
\hline$\overline{\mathrm{A}}$ & Obras provisionales & 15 & 9 & 4,403 & 5,283 & 6 & 146.8 \\
\hline $\mathrm{B}$ & Trabajos preliminares & 10 & 5 & 5,788 & 6,889 & 5 & 220.2 \\
\hline $\mathrm{C}$ & Movimiento de tierras & 5 & 3 & 17,744 & 20,947 & 2 & $1,601.3$ \\
\hline $\mathrm{D}$ & Cimentación & 12 & 7 & 88,552 & 91,655 & 5 & 620.6 \\
\hline $\mathrm{E}$ & Columnas y vigas & 10 & 6 & 98,292 & 107,596 & 4 & $2,326.1$ \\
\hline $\mathrm{F}$ & Instalaciones sanitarias & 15 & 10 & 29,172 & 34,361 & 5 & $1,037.7$ \\
\hline $\mathrm{G}$ & Instalaciones eléctricas & 13 & 7 & 14,327 & 16,450 & 6 & 353.8 \\
\hline $\mathrm{H}$ & Muros & 15 & 8 & 40,996 & 44,776 & 7 & 540.0 \\
\hline I & Revestimiento & 7 & 4 & 46,048 & 52,332 & 3 & $2,094.6$ \\
\hline $\mathrm{J}$ & Instalación de máquinas & 20 & 13 & 303,100 & 318,255 & 7 & $2,165.0$ \\
\hline K & Techo & 17 & 10 & 32,073 & 38,488 & 7 & 916.4 \\
\hline $\mathrm{L}$ & Acabados & 15 & 8 & 115,442 & 126,858 & 7 & $1,630.8$ \\
\hline & & & & 795,937 & 863,889 & & \\
\hline
\end{tabular}

La reducción máxima en días $M_{i}$ y el costo de reducir en soles por día $K_{i}$ también son mostrados en la Tabla 5.

En el cálculo de la reducción del tiempo de finalización del proyecto, se construye un modelo de programación lineal teniendo como función objetivo minimizar el costo total adicional por reducción del tiempo de finalización del proyecto tal como se plantea en (6).

Las restricciones estarán formadas por los cálculos de los tiempos de finalización más temprana de cada actividad, el tiempo de finalización más temprana de la 
última actividad en terminar, en este caso será $X_{L}$, y las del tiempo de reducción de cada actividad $i$, tal como se plantea en (7), (8) y (9) respectivamente, sin olvidar las restricciones de no negatividad de las variables $X_{i}$ e $Y_{i}$ como se establece en (10).

La solución del problema dependerá del valor del tiempo de finalización del proyecto $T_{\text {proy }}$, es por ello que existirá $n$ modelos para $n$ valores de $T_{\text {proy }}^{\text {proy }}$ que le demos en (8).

La Tabla 6 muestra las soluciones al problema de reducir el tiempo de finalización del proyecto $T_{\text {proy }}$ desde 95 a 55 días.

Tabla 6. Resumen de costos totales por reducción del tiempo de finalización del proyecto de conservas de pescado.

\begin{tabular}{|c|c|c|c|c|c|c|c|c|c|c|c|c|c|c|}
\hline \multirow{2}{*}{$\begin{array}{l}\text { Tiempo de finalización } \\
\text { del proyecto }\left(T_{\text {proy }}\right) \text { en } \\
\text { días }\end{array}$} & \multirow{2}{*}{$\begin{array}{c}\text { Costo adicional } \\
\text { por reducción } \\
(\mathrm{S} / .)\end{array}$} & \multirow{2}{*}{$\begin{array}{c}\text { Costo total } \\
(\mathrm{S} / .)\end{array}$} & \multicolumn{12}{|c|}{ Tiempo de reducción $Y_{i}$ de la actividad $i$ en días } \\
\hline & & & A & $\mathrm{B}$ & $\mathrm{C}$ & $\mathrm{D}$ & $\mathrm{E}$ & $\mathrm{F}$ & $\mathrm{G}$ & $\mathrm{H}$ & $\mathrm{I}$ & $\mathrm{J}$ & $\mathrm{K}$ & $\mathrm{L}$ \\
\hline 96 & 0 & 795,937 & & & & & & & & & & & & \\
\hline 95 & 147 & 796,084 & 1 & & & & & & & & & & & \\
\hline 94 & 293 & 796,230 & 2 & & & & & & & & & & & \\
\hline 93 & 440 & 796,377 & 3 & & & & & & & & & & & \\
\hline 92 & 587 & 796,524 & 4 & & & & & & & & & & & \\
\hline 91 & 733 & 796,670 & 5 & & & & & & & & & & & \\
\hline 90 & 1,100 & 797,037 & 6 & 1 & & & & & & & & & & \\
\hline 89 & 1,640 & 797,577 & 6 & 1 & & & & & & 1 & & & & \\
\hline 88 & 2,180 & 798,117 & 6 & 1 & & & & & & 2 & & & & \\
\hline 87 & 2,720 & 798,657 & 6 & 1 & & & & & & 3 & & & & \\
\hline 86 & 3,260 & 799,197 & 6 & 1 & & & & & & 4 & & & & \\
\hline 85 & 3,800 & 799,737 & 6 & 1 & & & & & & 5 & & & & \\
\hline 84 & 4,340 & 800,277 & 6 & 1 & & & & & & 6 & & & & \\
\hline 83 & 4,880 & 800,817 & 6 & 1 & & & & & & 7 & & & & \\
\hline 82 & 5,501 & 801,438 & 6 & 1 & & 1 & & & & 7 & & & & \\
\hline 81 & 6,121 & 802,059 & 6 & 1 & & 2 & & & & 7 & & & & \\
\hline 80 & 6,742 & 802,679 & 6 & 1 & & 3 & & & & 7 & & & & \\
\hline 79 & 7,363 & 803,300 & 6 & 1 & & 4 & & & & 7 & & & & \\
\hline 78 & 7,983 & 803,920 & 6 & 1 & & 5 & & & & 7 & & & & \\
\hline 77 & 8,900 & 804,837 & 6 & 1 & & 5 & & & & 7 & & & 1 & \\
\hline 76 & 9,816 & 805,753 & 6 & 1 & & 5 & & & & 7 & & & 2 & \\
\hline 75 & 10,732 & 806,670 & 6 & 1 & & 5 & & & & 7 & & & 3 & \\
\hline 74 & 11,649 & 807,586 & 6 & 1 & & 5 & & & & 7 & & & 4 & \\
\hline 73 & 12,565 & 808,502 & 6 & 1 & & 5 & & & & 7 & & & 5 & \\
\hline 72 & 13,482 & 809,419 & 6 & 1 & & 5 & & & & 7 & & & 6 & \\
\hline 71 & 14,398 & 810,335 & 6 & 1 & & 5 & & & & 7 & & & 7 & \\
\hline 70 & 16,000 & 811,937 & 6 & 1 & 1 & 5 & & & & 7 & & & 7 & \\
\hline 69 & 17,601 & 813,538 & 6 & 1 & 2 & 5 & & & & 7 & & & 7 & \\
\hline 68 & 19,232 & 815,169 & 6 & 1 & 2 & 5 & & & & 7 & & & 7 & 1 \\
\hline 67 & 20,863 & 816,800 & 6 & 1 & 2 & 5 & & & & 7 & & & 7 & 2 \\
\hline 66 & 22,494 & 818,431 & 6 & 1 & 2 & 5 & & & & 7 & & & 7 & 3 \\
\hline 65 & 24,125 & 820,062 & 6 & 1 & 2 & 5 & & & & 7 & & & 7 & 4 \\
\hline 64 & 25,755 & 821,693 & 6 & 1 & 2 & 5 & & & & 7 & & & 7 & 5 \\
\hline 63 & 27,386 & 823,323 & 6 & 1 & 2 & 5 & & & & 7 & & & 7 & 6 \\
\hline 62 & 29,017 & 824,954 & 6 & 1 & 2 & 5 & & & & 7 & & & 7 & 7 \\
\hline 61 & 31,112 & 827,049 & 6 & 1 & 2 & 5 & & & & 7 & 1 & & 7 & 7 \\
\hline 60 & 33,207 & 829,144 & 6 & 1 & 2 & 5 & & & & 7 & 2 & & 7 & 7 \\
\hline 59 & 35,655 & 831,592 & 6 & 1 & 2 & 5 & & & 1 & 7 & 3 & & 7 & 7 \\
\hline 58 & 38,335 & 834,272 & 6 & 1 & 2 & 5 & 1 & & 2 & 7 & 3 & & 7 & 7 \\
\hline 57 & 41,015 & 836,952 & 6 & 1 & 2 & 5 & 2 & & 3 & 7 & 3 & & 7 & 7 \\
\hline 56 & 43,695 & 839,632 & 6 & 1 & 2 & 5 & 3 & & 4 & 7 & 3 & & 7 & 7 \\
\hline 55 & 47,412 & 843,349 & 6 & 1 & 2 & 5 & 4 & 1 & 5 & 7 & 3 & & 7 & 7 \\
\hline
\end{tabular}

En cada solución al problema se encontraron las actividades que se deben de reducir y en cuanto, representados por la variable $Y_{i}$. La única actividad que no tuvo reducción en su tiempo fue la J, instalación de máquinas, debido a que tuvo un costo de reducción por día muy alto igual a S/. 2,165 y a la interrelación que hay entre las actividades. 
Enero - Junio 2016

Se puede observar que el costo total es igual al costo adicional por reducción más el costo total a tiempo normal.

En la reducción de 96 a 55 días hubo un incremento de $\mathrm{S} / .47,412$, teniendo como única actividad que no tiene reducción la $\mathrm{J}$, que es la instalación de máquinas.

\section{Conclusiones}

Utilizar la programación lineal en la formulación y solución a los problemas de acortamiento de los tiempos de finalización de proyectos permite encontrar la solución óptima a las variables tiempo de finalización más temprano $X_{i}$ y tiempo de reducción $Y_{i}$ de cada actividad $i$. El límite del acortamiento del tiempo de finalización del proyecto está dado por la reducción máxima del tiempo $t_{i}{ }_{i}$ y la relación de predecesoras inmediatas que hay entre las actividades. Con el modelo de programación lineal se puede acortar el tiempo de finalización del proyecto de 96 días hasta 55 días, con un costo adicional mínimo de $\mathrm{S} /$. 47,412, obteniéndose un costo total de S/. 843,349.

\section{Literatura citada}

Anderson, D; Sweeney, D; Williams, T. 2004. Métodos cuantitativos para los negocios. México D.F., México. Thomson. $822 \mathrm{p}$.

Hillier F; Lieberman G. 2006. Introducción a la investigación de operaciones. México D.F., México. McGraw-Hill. 1061 p.

Mehrotra, K; Chai, J; Pillutla, S. 1996. A study of approximating the moments of the job completion time in PERT networks. Journal of Operations Management 14(3): 227-289.

Render, B; Stair R; Hanna M. 2006. Métodos cuantitativos para los negocios. Naucalpan de Juárez, México. Pearson Educación. 731 p.

Taha H. 2004. Investigación de operaciones. Naucalpan de Juárez, México. Pearson Educación. 830 p.

Winston W; 2005. Investigación de operaciones: aplicaciones y algoritmos. México D.F., México. Thomson. $1418 \mathrm{p}$. 